\title{
What makes Lymphadenopathy in Patients with Autoimmune Thyroiditis Suspicious for Malignancy?
}

Authors

Affiliation

\author{
C. Bouter, B. Meller, J. Meller, C. O. Sahlmann
}

Depatment of Nuclear Medicine, Universitiy Medical Center (UMG) Goettingen, Goettingen, Germany

\author{
Key words \\ - thyroid \\ - lymph nodes \\ non-hodgkin lymphoma
}

received 20.07.2015

first decision 09.10.2015

accepted $\quad 17.11 .2015$

\section{Bibliography \\ DOI http://dx.doi.org/ 10.1055/s-0035-1569376 \\ Exp Clin Endocrinol Diabetes Rep 2016; 3: e1-e7 \\ (C) J. A. Barth Verlag in Georg Thieme Verlag KG Stuttgart · New York} ISSN 2196-7407

Correspondence

\section{Dr. C. Bouter}

Department of Nuclear Medicine

Centre for Radiology University Medical Center (UMG) Goettingen

Goettingen

Robert-Koch-Str. 40

37075 Goettingen

Germany

Tel.: + 49/551/398511

Fax: $+49 / 551 / 398526$

caroline.bouter@med.uni-

goettingen.de

\section{License terms}

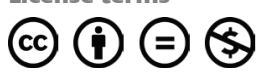

\section{Abstract \\ $\nabla$}

Lymphadenopathy in Robbins level II-IV and VI is common in autoimmune thyroiditis but the diagnostic strategy of a distinct lymphadenopathy exceeding the known features in autoimmune thyroiditis patients is unknown. The aim of this study was to determine how the extent of cervical lymphadenopathy in autoimmune thyroiditis affects the diagnostic management.

The study comprises one index-patient with autoimmune thyroiditis and distinct suspicious lymphadenopathy in all cervical levels as well as retroclavicular. In addition 10 patients with autoimmune thyroiditis and distinct suspicious lymphadenopathy limited to level VI were evaluated.

Findings of high resolution ultrasound, fine-needle aspiration cytology, serological testing and clonal analysis are reported here. Further diagnostics of the index-patient included histology, immunohistochemistry, bcl-2-expression analysis and PET/CT.

\section{Introduction \\ $\nabla$}

Autoimmune thyroiditis (AIT) is one of the most common chronic autoimmune diseases affecting up to $2 \%$ of the world's population [1]. AIT is characterized by T-cell mediated diffuse lymphocyte infiltration of the thyroid causing destruction and functional impairment of thyroid tissue that leads to hypothyroidism in $30 \%$ of cases [2,3].

Diagnosis of AIT is based on ultrasound and laboratory proof of elevated antibodies against thyroid peroxidase and/or thyroglobulin. Ultrasound typically displays a diffuse hypoechoic pattern of the thyroid parenchyma [2].

An increased prevalence of enlarged cervical lymphatic nodes was reported during the last years in the literature. Serres-Créixams et al.
The index-patient showed distinct lymphadenopathy in level I-VI and retroclavicular. Lymph nodes did not display any sonographic malignancy criteria. Molecular analysis and immunohistochemistry revealed monoclonal CD10- and CD20-positive, Bcl-2 expressing follicular B-cells confirming the diagnosis of a follicular B-cell Non-Hodgkin lymphoma.

10 additional patients with limited lymphadenopathy showed typical features of autoimmune thyroiditis and lymph nodes did not display sonographic malignancy criteria in all cases. Further tests excluded lymph node malignancies.

Extensive diagnostics in autoimmune thyroiditis and distinct cervical lymphadenopathy is crucial. Whereas limited lymphadenopathy in AIT was proven benign, extensive lymphadenopathy exceeding the known amount and/or spreading to retroclavicular/mediastinal compartments is suspicious for malignancy. Even in the absence of sonographic malignancy criteria further tests have to be performed.

focused especially on LAP in in Robbins level VI (RL VI) [4]. Data was prospectively confirmed by our group, which first described an elevated incidence of LAP in RL II-IV in AIT patients compared with controls [3]. Brancato et al. [5] and Jones et al. [6] added further data, which confirmed these earlier observations with a decent amount of patients. Summarizing the current knowledge, it is presumed that cervical lymphadenopathy (LAP) in RL II-IV and VI is related to the autoimmune process and might be even pathognomic for AIT in RL VI, when other otolaryngologic malignancies can be excluded. However, there is no data about the diagnostic strategy if LAP in AIT patients exceeds the known amount and/or spreads to mediastinal compartments. Therefore, the aim of the study was to compare limited and extensive LAP in AIT patients in order to deter- 
mine when the diagnostic management in AIT patients with LAP has to change.

\section{Subjects and Methods \\ $\nabla$}

\section{Index-patient}

A 38-year-old male patient with AIT and RL I-VI LAP presented in our unit. Evaluation of the thyroid and cervical LN was carried out with high resolution ultrasound (HD11 XE Ultrasound System; fitted with a $5-13 \mathrm{MHz}$ linear transducer; Philips Deutschland, Hamburg, Germany) in our institution. Fine-needle aspiration cytology (FNAC) of the right thyroid lobe and one LN in RL IV was performed. Thyroid parameters, a white blood cell count and the determination of CRP were done and infection with Borellia Burgdorferi, Cytomegaly virus (CMV) Epstein Barr Virus (EBV), Herpes simplex virus (HSV), Adenovirus, Coxsackie virus, Toxoplasmosis and Human immunodeficiency virus (HIV) were serologically evaluated. Tissue samples were paraffin-embedded and stained with hematoxylin and eosin. Immunohistochemistry was performed with antibodies against CD3, CD5, CD10, CD20, CD23, bcl-2 and Cyclin-D1. For clonality analysis the IGH Gene Clonality Assay (IdentiClone ${ }^{\mathrm{TM}}$, InVivoScribe Product Group) with primers Fr2A, Fr3A, LJH and VLJH was used after standard protocol provided by the manufacturer. Amplification of the target DNA was performed with the universal thermocycler program.

Fluorescent-in-situ-hybridization (FISH) for BCL2 was carried out with dual-color, break-apart probes.

PET/CT was performed after a common scheme used in our facility. The Patient was fasting at least $6 \mathrm{~h}$ before examination. Blood glucose was measured before the injection of 18F-FDG, a blood glucose level under $120 \mathrm{mg} / \mathrm{dl}$ was verified. After the injection the patient had to being orally hydrated. PET/CT was performed $60 \mathrm{~min}$ after application of [ $\left.{ }^{18} \mathrm{~F}\right] \mathrm{FDG}$ on a Philips Gemini TF16 PET/CT-scanner (Philips Medical Systems, Cleveland, OH, USA) with a $196 \times 196$ matrix with $4 \mathrm{~mm}$ slice thickness and lowdose-CT with a $512 \times 512$ matrix with $2 \mathrm{~mm}$ slice thickness for attenuation correction (CTAC-SG algorithm). The reconstruction was performed using the LOR-TF-RAMLA ("BLOB-OS-TF") algorithm (Surti). Images were evaluated multiplane frontally, sagittal and axillary scanning for regions of focally increased tracer uptake. Abnormal FDG uptake was defined as accumulation of the tracer above the physiological uptake.

\section{AIT patients with LAP}

In continuance to earlier studies in which marked LAP in RL VI was the most prominent and lymphoma suspicious feature in AIT patients, we selected 10 patients with distinct suspicious LAP in this RL for an extensive workup. We retrospectively evaluated results of those 10 autoantibody positive (anti-TPO) patients (female: $n=9$, male: $n=1$; median age 33 years; range: 18-62 years) here. Patients were evaluated for enlarged LN by high resolution ultrasound (Siemens ACUSON Antares ultrasound scanner fitted with a $5-13 \mathrm{MHz}$ linear transducer; Siemens Deutschland, Erlangen, Germany) in our institution during 2013-2014. Cut-off for enlarged LN were used as follows: $5 \mathrm{~mm}$ in RLVI, $>1 \mathrm{~cm}$ in RL I and $>2 \mathrm{~cm}$ in RL II-V. The cut offs were chosen in accordance to data provided in the seminal work of Koischwitz for non-carcinoma patients. We took into account that LN in RL VI in healthy controls are a very rare finding and therefore used a relatively small diameter for defining a patho- logical LN in RL VI according to a sensitive approach. Submandibular LN up to $2 \mathrm{~cm}$ are common findings in non-AIT patients and the same applies to RL II-V and a cut-off of $1 \mathrm{~cm}$.

FNAC of lymphatic nodes was performed in 9 patients. Clonality analysis was performed by IGH Gene Clonality Assay (IdentiClone $^{\mathrm{TM}}$, InVivoScribe Product Group). Primers Fr2A, Fr3A, LJH and VLJH were used. Thyroid parameters, a white cell blood count and the determination of CRP were performed in all patients. Infection with Borellia Burgdorferi and Cytomegaly virus (CMV) were serologically evaluated in all patients. 5 patients were additionally screened for infections with Epstein Barr Virus (EBV), Herpes simplex virus (bHSV), Adenovirus, Coxsackie virus, Toxoplasmosis and Human immunodeficiency virus (HIV). A follow-up of at least 6 month was available in all patients.

\section{Results}

$\nabla$

\section{Index-patient}

A 38-year-old man recognized a cervical swelling and therefore presented for further evaluation of the thyroid. He did not complain about local afflictions or any symptoms of thyroid dysfunction. Furthermore, the patient did not report any signs of B symptoms and stated overall well-being. His medical history did not provide thyroid disorders. However, the patient reported a resection of a cervical lymph node in his childhood/adolescence but could not recall about further details, histological evaluation might have shown anthracosis or granulomatous changes. Physical examination revealed nodular goiter and multiple palpable lymph nodes with medium consistency. On examination, the patient had normal weight, $78 \mathrm{~kg}$ measuring $184 \mathrm{~cm}$ in height and presented in a good general state of health.

Sonography showed a diffuse enlarged, suprasternal thyroid gland of $40 \mathrm{ml}$ with a heterogeneous hypoechoic texture, lobulation and signs of fibrosis without any suspicious nodules, diagnostic for autoimmune thyroid disease ( $\odot$ Fig. 1). Multiple, oval-shaped lymph nodes appeared in RL I, II, III, IV, V and VI on both sides of the neck expanding to the retroclavicular compartment. Longitudinal/transversal quotient was normal in these LN and LN did not show irregular margins, atypical hyperperfusion, microcalcification or cystic degeneration. Some of these LN showed a facet formation by abutting each other. The largest lymph node had a longitudinal diameter of $3.5 \mathrm{~cm}$. Follow-up examination 6 month later showed constant findings.

Laboratory analysis revealed elevated thyroglobulin antibodies and a subclinical hypothyroidism. Along with a typical cytology the diagnosis of Hashimoto's thyroiditis was confirmed.

It remained unclear whether the LAP was associated with the autoimmune thyroid disease (Sahlmann et al. 2012), occult thyroid lymphoma, thyroid cancer or was the manifestation of an independent infection with adenotropic viruses or extratyroidal lymphoma. Therefore further tests were performed.

Serology revealed positive anti-Coxsackievirus antibodies, pointing to a currently undergone infection or persistency of antibodies. A currently occurred infection with Adoenovirus could not be excluded due to slightly elevated Adenovirus-antibodies.

Cytological evaluation showed a lymphocytic inflammation of thyroid tissue corresponding to a chronic inflammation in the context of Hashimoto's disease. 

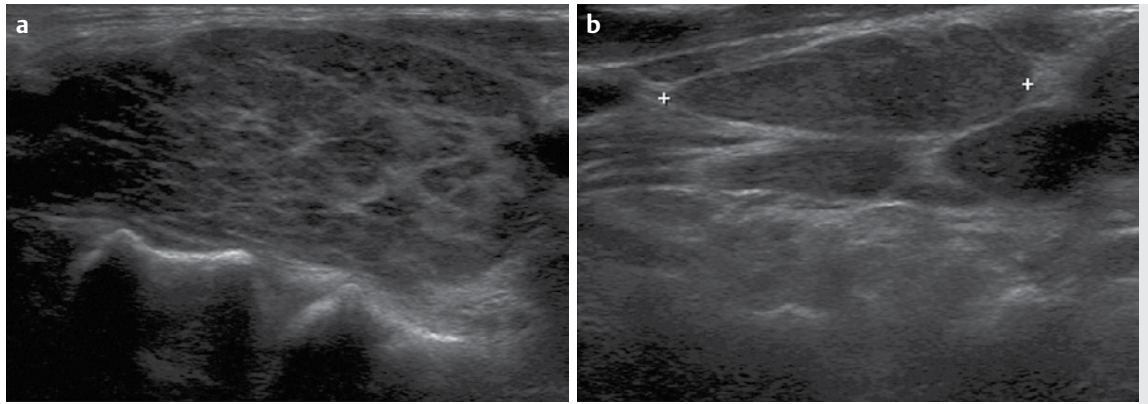

Fig. 1 Utrasound of the index-patient shows thypical features of AIT and distinct cervical LAP. a Logitudinal view of right thyroid lobe. b Cervical lymph node, longitudinal diamenter (between $(+)): 2.92 \mathrm{~cm}$. c Cervical lymph nodes show facet formation. $\mathbf{d}$ Doppler sonography of cervical lymph nodes indicating hilar vessels.
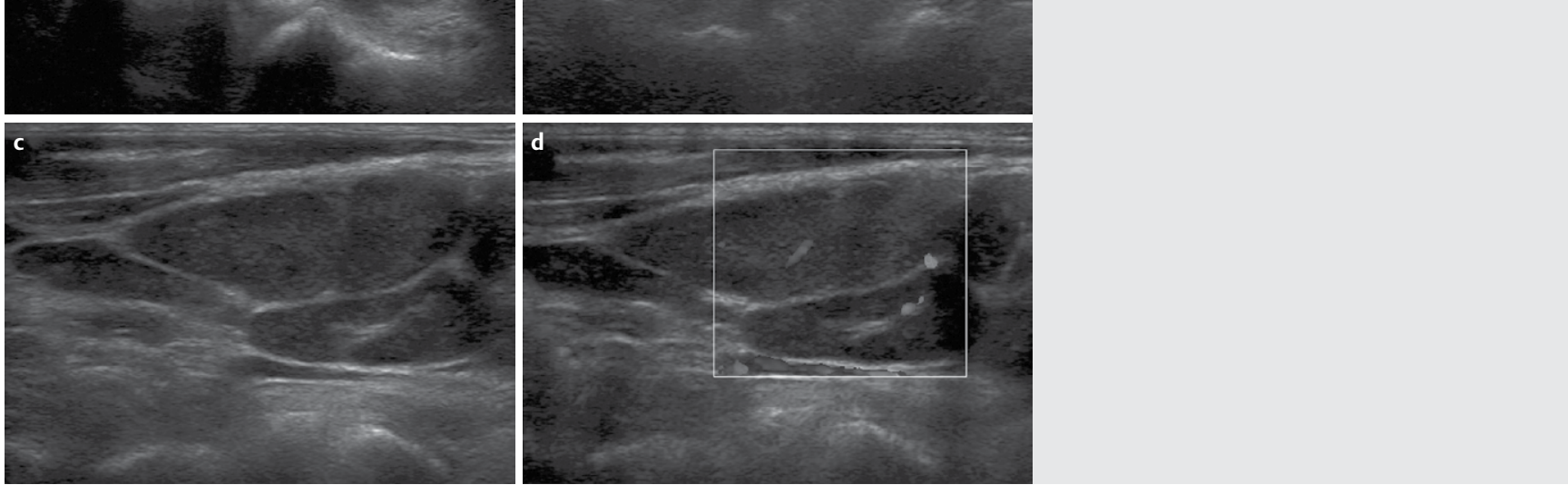

Molecular pathology revealed a monoclonal rearrangement pattern of IgH within thyroid tissue punctuate as well as within samples of cervical lymph nodes suspecting a malignant neoplasia. Further histological examination of a resected cervical lymph node showed hyperplasic tissue whereby immunhistochemistry revealed a CD10 positive, bcl-2 expressing B-cell population establishing the diagnosis of a B-cell non-Hodgkin lymphoma (NHL) of a follicular type. A further translocation analysis by fluorescent in situ hybridization (FISH) revealed a bcl-2 translocation 18q21 verifying the diagnosis of a NHL. Additional performed Fluorescence-activated cell sorting (FACS) analysis confirmed a washout of lymphoma cells in the peripheral blood. NHL was staged to IVA as a extranodular manifestations as well as distinct LN involvement.

Positron emission tomography with $\left[{ }^{18} \mathrm{~F}\right] \mathrm{FDG}$ revealed an involvement of cervical, mediastinal, mesenterial, axillary, paraaortic, iliac and inguinal lymph nodes and pulmonal involvement by the NHL ( $\odot$ Fig. 2). Furthermore, a diffuse FDG-uptake in the thyroid was seen which can occur in the context of AIT as well as a manifestation of the thyroidal involvement of the NHL.

\section{AlT patients with LAP}

These patients were selected for a marked and lymphoma suspicious LAP in RL VI. All 10 patients showed the typical features of AIT on ultrasound ( Fig. 3) and had 2-9 (in total 79 documented) lymphatic nodes $>5 \mathrm{~mm}$ in RL VI (Median transversal diameter: $1,4 \mathrm{~cm}$; range: $0.9-2.3 \mathrm{~cm})$. Enlarged $\mathrm{LN}$ in other compartments beyond normal range (more than $5 \mathrm{LN}>5 \mathrm{~mm}$ or single $\mathrm{LN}>1 \mathrm{~cm}$ in RL I and $>2 \mathrm{~cm}$ in RL II-V) were not present. Enlarged RL VI LN had an oval shape in 60 LN (76\%) and were rounded in $19 \mathrm{LN}$ (24\%). A detectable hilum was present in only 45 LN (57\%), echogenicity was hypoechoic in 71\% (56 LN) and marked isoechoic in 29\% (23 LN), margins were sharp and LN did not show any structural changes like intranodal necrosis, reticulation and calcification in all cases. In some patients LN abutted each other showing a facet formation ( $\bullet$ Fig. 3c, d). FNAC of the LN was performed in 9 cases revealing reactive lymphoid hyperplasia in 9 patients. Clonality was excluded in these patients. White cell blood count and CRP levels were within the normal range in all patients. Actual infections with CMV, EBV, HSV, Ade- novirus, Coxsackie virus, Toxoplasmosis, HIV and Borellia Burgdorferi were ruled out in all patients.

During follow-up, LAP persisted in 8 patients and regressed in 2 patients. LAP was still limited to RL VI.

\section{Discussion \\ $\nabla$}

The reported case displays the importance of differentiating cervical lymph nodes in patients with AIT and distinct LAP. Differential diagnosis included inflammatory activity in the context of AIT, metastases of an occult microcarcinoma or primary lymphoma of the thyroid as well as extrathyroidal lymphoma.

Earlier studies strongly suggest that a specific pattern of lymphatic involvement exists in AIT [3-6]. Regarding the sites of LAP, enlarged lymph nodes of the cricothyroidal and pretracheal/infrathyroidal compartment (RL VI) were almost selectively found in AIT-patients by Sahlmann et al., Serres-Creîxams et al. and Akbaba et al. and have to be considered as pathognomonic for the disease in the absence of head and neck cancer $[3,4,7]$. Furthermore, Serres-Creîxams et al. suggested an association of paratracheal LAP and inflammatory activity of AIT. Sahlmann et al., Brancato et al. and Jones et al. reported a significant increase in hyperplastic LN in RL II-IV [3,4,6]. The 10 AIT patients in this study were selected for their distinct and lymphoma-suspicious LAP in RL VI and are not representative for the whole spectrum of LN distribution patterns in AIT. Our extensive molecular work-up confirm the earlier findings of Sahlmann et al., that a limited distinct LAP in RL VI usually represents benign LAP.

However, Brancato et al. and Jones et al. were not able to demonstrate a significantly increased LAP in RL VI $[5,6]$. Brancato et al. used an arbitrary cut-off of $1 \mathrm{~cm}$ in the long-axis diameter grossly underestimating the prevalence of LAP in RL VI in which usually smaller bunched LN are common findings in ultrasound. Findings of Jones et al. confirmed that enlarged LN in RL VI are very rare in patients without AIT. However, the low amount of enlarged LN in RL VI in AIT patients in that study remains unclear and may be explained by a different clinical presentation of AIT patients in the United States compared to central Europe with a 

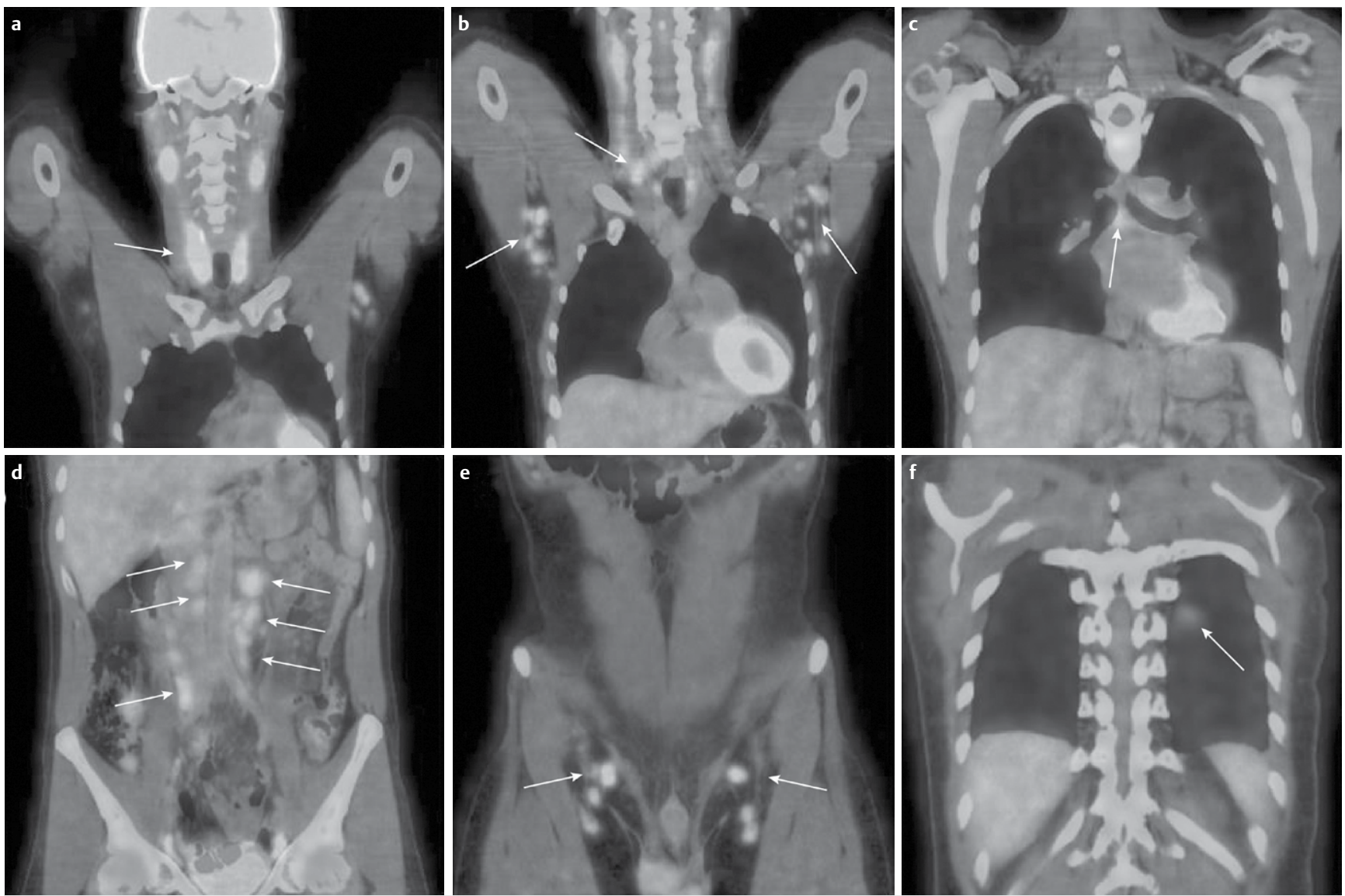

Fig. $2\left[{ }^{18} \mathrm{~F}\right] \mathrm{FDG}$ PET/CT of the index-patient displays the extend of lymph node involvement. a Thyroidal uptake. b Involvement of cervical and axillary LN c Involvement of mediastinal LN. d Involvement of paraaortic LN. e Involvement of iliac LN. b Pulmonal manifestation.
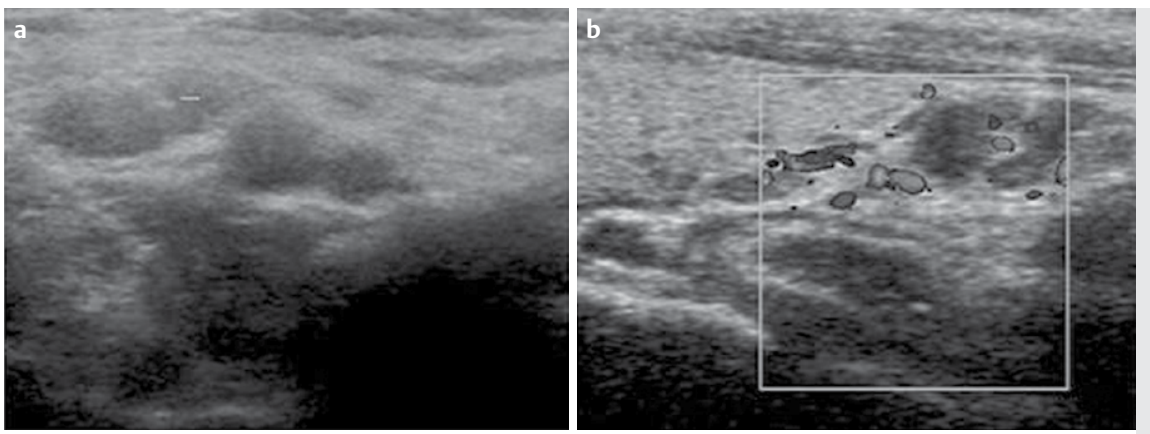

Fig. 3 Sonographic features of LAP in AIT patients. a Enlarged benign lymph node of one AIT patient in our study. $\mathbf{b}$ Hyperperfusion of a benign cervical lymph node. $\mathbf{c}$ and $\mathbf{d}$ Facet formation was seen in some of the 10 AIT patients, 2 patients are exemplary shown here.
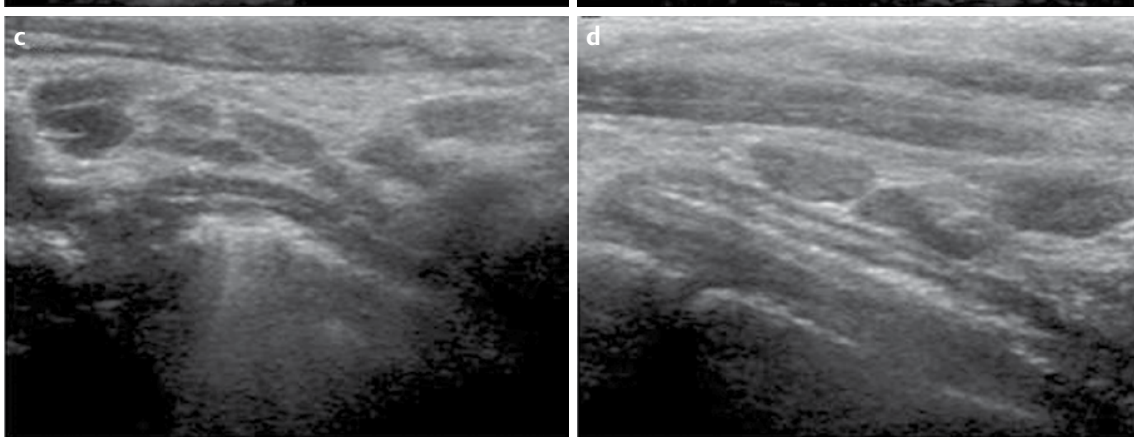

higher prevalence of a hypertrophic nodular form of AIT compared to a majority of cases without a goiter in central Europe. The described index-patient also showed a cervical LAP. However, in this case LAP was very distinct with bunched LN in all cervical levels expanding to the retroclavicular compartment.
Lymph nodes did not show sonographic signs of malignancy like structural changes, irregular margins or microcalcification (B scan criteria) which did not differ from AIT patients with distinct benign LAP. In comparison to previous studies reporting a higher incidence of enlarged LN in RL II-IV, the amount and dis- 
tribution pattern of enlarged $\mathrm{LN}$ in our index patient differed broadly to enlarged LN in the context of inflammation of AIT patients. Brancato et al. reported a mean total number of $\mathrm{LN}$ in RL II-IV between 0.7 and 2 per level and LN sizes (long axis diameter) between 13.3 and $15.9 \mathrm{~mm}$ in RL II-IV of AIT patients. In our index patient the amound of LN was much higher and LN appeared conspicuously bunched in RL I-V and therefore further examinations were initiated. Several possible diagnoses were evaluated and are discussed here.

Association of AIT and thyroid carcinoma has been previously reported but is still controversial. Lindsay et al. (1955) first described an association of AIT and papillary thyroid carcinoma [8]. Several groups found a high incidence of AIT in papillary thyroid carcinoma up to $44 \%[9,10]$. Data of our group show an incidence of $21 \%$ of AIT in thyroid carcinoma patients (Bouter et al., unpublished data). Chronic inflammation is supposed to be a risk factor for the development of malignancies. Guarino et al. (2010) stated a pro-tumorigenic function of inflammatory cells that might facilitate RET-oncogene rearrangement within thyroid cells [11]. RET- Mutations are thought to be essential in tumorgenesis [12]. Furthermore, cytokines and chemokines released by the inflammatory tissue might activate anti-apoptotic pathways leading to a resistance against oncogene-induced apoptosis and survival of thyroid cells expressing the RET-protooncogene. In patients with thyroid carcinomas lymph node metastases are very common. Up to $70 \%$ of patients show cervical LN metastases [13]. Even patients with microcarcinomas $\leq 10 \mathrm{~mm}$ that are difficult to localize with ultrasound (especially in diffuse hypoechoic glands of AIT patients) frequently show LN metastases. Wada et al. (2003) reported the central and ipsilateral lateral compartments (RL VI and RL II-IV) as common sites of metastasis whereas the posterior triangle (RL V) is rarely involved [14]. Henry et al. (1998) described the central compartment (RL VI) as primary zone of thyroid carcinoma metastasis whereby the upper thyroid-pole is suggested to drain into the ipsilateral lateral compartment (RL II/III) [15]. Furthermore, several groups suggest sentinel node detection and biopsy to study sites of lymphatic drainage lowering the risk of occult metastasis $[16,17]$. Ultrasound combined with Doppler sonography of cervical lymph nodes is an important and well established diagnostic procedure in the assessment of lymph node metastases. B scan criteria as size, shape, intranodal necrosis, calcification or hyperperfusion help differentiating between malign and benign cervical lymph nodes. However, in our index case sonographic B scan features besides enlargement of lymph nodes were not present. Microcarcinomas of the thyroid can be hard to detect within ultrasound as changes of the ultrastructure with a diffuse hypoechogenity of the gland makes a differentiation of small hypoechoic lesions very difficult. In this case, the extent and the sonographic appearance of involved LN as well as sonographic findings within the thyroid spoke against a thyroid carcinoma as the cause of the LAP.

\section{Sonographic changes of lymph nodes are also described in lymphomatous lymph nodes [18]}

Ahuja et al. (2008) described sonographic features of involved cervical lymph nodes in lymphomas not differing from metastases in size, shape, echogenity and hilus [19]. However, calcification and intranodal necrosis is uncommon in lymhomatous lymph nodes distinguishing from metastatic lymph nodes. Furthermore, unlike metastases vascular distribution includes both peripheral and hilar vessels. Some LN of the index-patient did show hyperperfusion peripheral as well as hilar. However, this distribution pattern has also been described by Sahlman et al. (2012) in LN of AIT patients with distinct benign LAP [3]. These findings display that a differentiation between malignity and benignity based on the vascular distribution alone is not sufficient. Furthermore, cervical LN in B-cell lymphomas often show a facet formation with LN abutting each other [20]. This facet sign was also partially seen in our index-patient ( $\boldsymbol{O}$ Fig. $\mathbf{1 b}, \mathbf{c}$ ). However, facet formations also occur in AIT-patients with distinct LAP. Several patients within our study with negative clonality analysis in FNAC showed a facet formation sonographically, pointing out that relying on this sonographic feature is not sufficient as well.

Primary lymphoma of the thyroid (PTL) is a rare disease representing less than $5 \%$ of thyroid malignancies or extranodal lymphomas [21]. Association of primary thyroid lymphomas with AIT has been reported in the literature [22,23]. Incidence of AIT in thyroid lymphoma patients is reported up to $100 \%$ in several studies [24-26]. Chronic inflammation in the course of autoimmune diseases is assumed a risk factor for development of a thyroid lymphoma. Progress of AIT into lymphoma occurs in about $0.5 \%$ of cases and develops in an average of 25 years after the onset of AIT [27]. Clinically, thyroid lymphoma presents as a rapidly growing mass in the neck. PTL can metastasize in cervical lymph nodes determining the prognosis of the disease [21]. The majority of primary thyroid lymphomas is mucosa-associated lymphoid tissue (MALT) or diffuse large cell lymphomas. Only $3-5 \%$ of cases are follicular lymphomas. Sonographic findings within the thyroid of PTL include single nodular masses or a multinodular goiter [28]. Although our index-patient presented with a goiter, he did not show signs of a rapidly growing mass in the thyroid and no nodular structures were seen within the thyroid tissue in ultrasound and therefore PTL was ruled out as differential diagnosis.

FNAC was performed in our index-patient showing a lymphocytic inflammation within the thyroid tissue. Clonality analysis displayed a monoclonal rearrangement of the variable region of the immunoglobulin heavy chain in the thyroid tissue using PCR. B-cell NHLs show monoclonality in up to $90 \%$ of cases and PCR using primers for regions of the heavy chain is widely used as a diagnostic tool in lymphoma patients $[29,30]$. Rearrangement of the immunoglobulin heavy chain gene is an early event in normal B-cell development leading to a polyclonal population of B-cells with unique rearrangement patterns. PCR analysis in these B-cell populations yields a heterogeneous picture [31]. In NHLs multiple genetic events lead to a progressive clonal expansion of B-cells lacking divers rearrangement patterns. These monoclonal B-cell populations display a homogeneous PCR-profile. Immunoglobulin heavy chain gene rearrangement assays can identify monoclonality and are thereby essential in differentiating a florid lymphocytic infiltrate and lymphoid malignancies. However, monoclonal rearrangement of B-cells can also occur in autoimmune diseases [32]. Several groups reported clonal B-cell populations within thyroid tissue samples of AIT patients in a vast minority of cases, but features are non-reproducible in multiple tissue samples [33-35]. Whether or not these monoclonal populations can evolve into a thyroid lymphoma remains unclear to date[33]. In our index case molecular analysis was a valuable tool to distinguish between a florid polyclonal lymphoid infiltrate and monoclonal lymphoma. Without performing the molecular analysis a differentiation would not have been possible. 


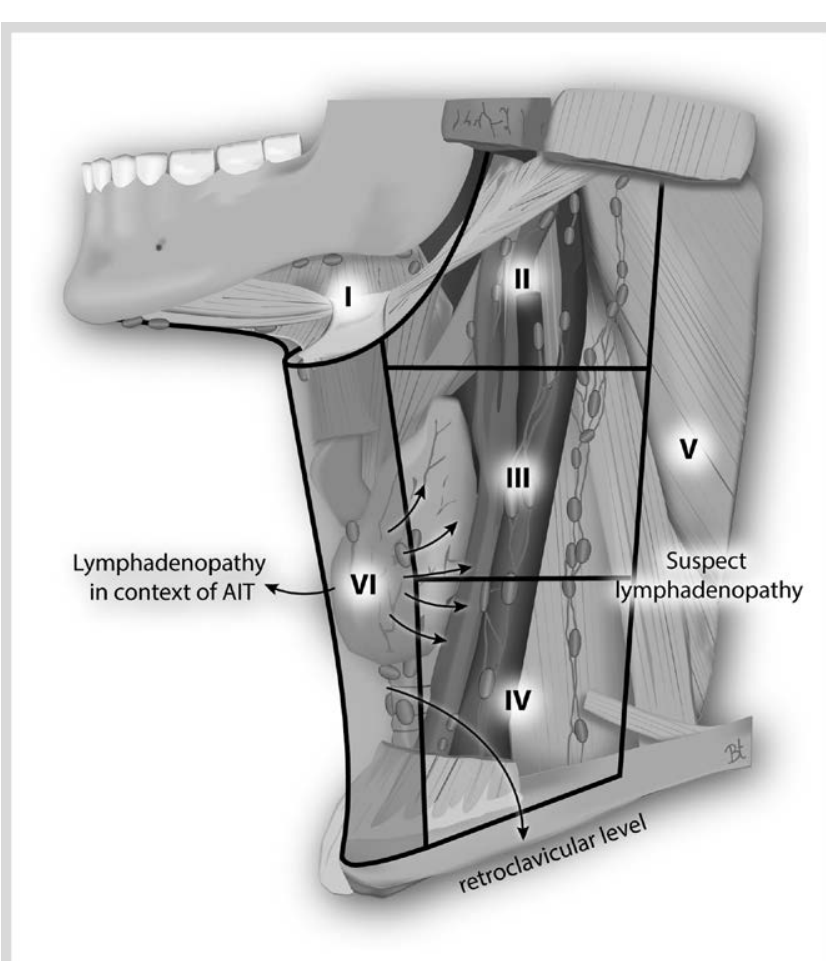

Fig. 4 Cervical lymph nodes can be divided into 6 levels [40]. RL VI (marked red) is supposed to be pathognomic for AIT. LAP that includes RL $\mathrm{I}-\mathrm{V}$ and the retroclavicular compartement has to be considered malignant and further tests have to be carried out. Figure was generated with Adobe Illustrator CS6 after Robbins et al. [40].

Furthermore, fluorescent in situ hybridization (FISH) detected a $\mathrm{Bcl}-2$ translocation in our index-patient. Bcl-2 translocation is detectable in $90 \%$ of follicular B-cell lymphomas within lymph nodes [36]. Bcl-2 translocation is assumed to be involved in the development of NHL. Translocation results in overexpression of the bcl-2 protein, a member of an anti-apoptotic protein family. However, primary extranodal follicular lymphomas often remain localized and mostly lack Bcl-2 translocation [37]. In our index case, in comparison with molecular pathology of the LN, findings within the thyroid mark an extranodal manifestation of the primary extrathyroidal lymphoma in the thyroid.

Follicular NHL represents the most common type of indolent lymphomas and the second most common type of all NHL [38]. Transformation to a diffuse large cell occurs in $10-70 \%$ of cases over time associated with rapid progression and extranodal disease [39]. As described above Clonality analysis helped differentiating between polyclonal lymphocytic infiltrate and B-cell lymphoma. Follicular lymphoma cells express CD 10, CD 19 and CD20 and overexpress Bcl-2 in almost all cases [36]. In our index-patient histology and immunostaining revealed CD10 and CD20 positive, Bcl-2 expressing follicular B-cells confirming the diagnosis of a follicular B-cell NHL, grade 1.

The described patient points out the difficulty of differentiating between benign and malign cervical LAP. Further tests revealing the NHL were performed only on the basis of the distribution and the visual appearance of the LAP beyond RL II-IV and RL VI and the expansion to the retroclavicular compartment. Common ultrasound malignancy criteria did not help distinguishing between benign and malign LAP in the AIT patient. Next to sonographic B scan criteria, the distribution of detectable lymph nodes seems to be a crucial factor in differentiating a cervical
LAP. Therefore, it can be stated that LAP exceeding RL II-IV and RL VI to the retroclavicular compartment, even though sonographic malignancy criteria are not present, is suspicious for a systemically malignant disease ( $\odot$ Fig. 4). Accurate distinction of the diagnosis of a cervical LAP is essential for further therapeutic management.

Extension of distinctly enlarged lymph nodes beyond RL VI to the retroclavicular compartment has to be considered malignant and therefore further diagnostics are important. Whereas previous studies reported a higher incidence of enlarged LN in RL II-IV the decisive parameter is the amount of enlarged LN and the distribution pattern. Therefore, not every patient with a certain amount of LN in other RLs besides RL VI has to be studied extensively. However, if a distinct LAP with bunched LN is present a malignant disease has to be considered. Certain criteria have to be included to differ between benign LAP in AIT, thyroid carcinoma, primary lymphoma of the thyroid and lymphoma of cervical lymph nodes. Next to ultrasound, diagnostics of extensive LAP should include serology testing for adenotropic viruses, FNAC with subsequent cytology and immunohistochemistry. Furthermore, molecular analysis is essential to display monoclonality proving a lymphoid malignancy. PET/CT can be useful to explore the extent of LN involvement besides the neck area.

\section{Conclusion}

$\nabla$

Common ultrasound malignancy criteria are insufficient in the differential diagnosis of a cervical LAP in AIT patients. Based on the extent of involved LN especially bunched LN and expansion to the retroclavicular compartment, next to a benign cause in the context of AIT, a malignant disease has to be considered and further tests have to be performed.

\section{Funding}

$\nabla$

This research did not receive any specific grant from any funding agency in the public, commercial or not-for-profit sector.

\section{Acknowledgements}

$\nabla$

We thank Orthrud Gerke for her administrative help.

Declaration of interest: The authors declare that there is no conflict of interest.

\section{References}

1 Wang C, Crapo LM. The epidemiology of thyroid disease and implications for screening. Endocrinol Metab Clin North Am 1997; 26: 189-218

2 Chistiakov DA. Immunogenetics of Hashimoto's thyroiditis. J Autoimmune Dis 2005; 2: 1

3 Sahlmann CO, Meller J, Siggelkow H, Homayounfar K, Ozerden M, Braune I. Patients with autoimmune thyroiditis. Prevalence of benign lymphadenopathy. Nuklearmedizin 2012; 51: 223-227

4 Serres-Créixams X, Castells-Fusté I, Pruna-Comella X, Yetano-Laguna V, Garriga-Farriol V, Gallardo-Agromayor E. Paratracheal lymph nodes: a new sonographic finding in autoimmune thyroiditis. J Clin Ultrasound 2008; 36: 418-421

5 Brancato $D$. Neck lymph nodes in chronic autoimmune thyroiditis: the sonographic pattern. Thyroid 2013; 173-177

6 Jones MR. The presentation of lymph nodes in Hashimoto's thyroiditis on ultrasound. Gland Surg 2015; 301-306 
7 Akbaba G. Changes in the Charactersitics of Paratracheal Lymph Nodes in Patients with Chronic Autoimmune Thyroiditis for Five Years Follow-up. Endocrine Society's 96th Annual Meeting and Expo, June 21-24, 2014 - Chicago. Chicago, USA 2014;

8 Lindsay S, Dailey ME. Malignant lymphoma of the thyroid gland and its relation to Hashimoto disease: a clinical and pathologic study of 8 patients. J Clin Endocrinol Metab 1955; 15: 1332-1353

9 Girardi FM, Barra MB, Zettler CG. Papillary thyroid carcinoma: does the association with Hashimoto's thyroiditis affect the clinical-pathological characteristics of the disease? Braz J Otorhinolaryngol 2014

$10 \mathrm{Kim}$ ES, Lim DJ, Baek KH, Lee JM, Kim MK, Kwon HS. Thyroglobulin antibody is associated with increased cancer risk in thyroid nodules. Thyroid 2010; 20: 885-891

11 Guarino V, Castellone MD, Avilla E, Melillo RM. Thyroid cancer and inflammation. Mol Cell Endocrinol 2010; 321: 94-102

12 Santoro M, Melillo RM, Carlomagno F, Fusco A, Vecchio G. Molecular mechanisms of RET activation in human cancer. Ann N Y Acad Sci 2002; 963: 116-121

13 Shaha AR, Shah JP, Loree TR. Patterns of nodal and distant metastasis based on histologic varieties in differentiated carcinoma of the thyroid. Am J Surg 1996; 172: 692-694

14 Wada N, Duh Q, Sugino K, Iwasaki H, Kameyama K, Mimura T. Lymph node metastasis from 259 papillary thyroid microcarcinomas: frequency, pattern of occurrence and recurrence, and optimal strategy for neck dissection. Ann Surg 2003; 237: 399-407

15 Henry JF, Gramatica L, Denizot A, Kvachenyuk A, Puccini M, Defechereux $T$. Morbidity of prophylactic lymph node dissection in the central neck area in patients with papillary thyroid carcinoma. Langenbecks Arch Surg 1998; 383: 167-169

16 Raijmakers PGHM, Paul MA, Lips P. Sentinel node detection in patients with thyroid carcinoma: a meta-analysis. World J Surg 2008; 32: 1961-1967

17 Dzodic R, Markovic I, Inic M, Jokic N, Djurisic I, Zegarac M. Sentinel lymph node biopsy may be used to support the decision to perform modified radical neck dissection in differentiated thyroid carcinoma. World J Surg 2006; 30: 841-846

18 Dudea SM, Lenghel M, Botar-Jid C, Vasilescu D, Duma M. Ultrasonography of superficial lymph nodes: benign vs. malignant. Med Ultrason 2012; 14: 294-306

19 Ahuja AT, Ying M, Ho SY, Antonio G, Lee YP, King AD. Ultrasound of malignant cervical lymph nodes. Cancer Imaging 2008; 8: 48-56 doi:10.1102/1470-7330.2008.0006

20 Gritzmann N, Koischwitz D. Ultrasound of the neck. J Otolaryngol 1993; 22: 315-320

21 Sakorafas GH, Kokkoris P, Farley DR. Primary thyroid lymphoma (correction of lympoma): diagnostic and therapeutic dilemmas. Surg Oncol 2010; 19: e124-e129

22 Hyjek E, Isaacson PG. Primary B cell lymphoma of the thyroid and its relationship to Hashimoto's thyroiditis. Hum Pathol 1988; 19: 1315-1326

23 Scholefield JH, Quayle AR, Harris SC, Talbot CH. Primary lymphoma of the thyroid, the association with Hashimoto's thyroiditis. Eur J Surg Oncol 1992; 18: 89-92
24 Anscombe AM, Wright DH. Primary malignant lymphoma of the thyroid - a tumour of mucosa-associated lymphoid tissue: review of seventy-six cases. Histopathology 1985; 9: 81-97

25 Matsuzuka F, Miyauchi A, Katayama S, Narabayashi I, Ikeda H, Kuma K. Clinical aspects of primary thyroid lymphoma: diagnosis and treatment based on our experience of 119 cases. Thyroid 1993; 3: 93-99

26 Kato I, Tajima K, Suchi T, Aozasa K, Matsuzuka F, Kuma K. Chronic thyroiditis as a risk factor of B-cell lymphoma in the thyroid gland. Jpn J Cancer Res 1985; 76: 1085-1090

27 Pedersen RK, Pedersen NT. Primary non-Hodgkin's lymphoma of the thyroid gland: a population based study. Histopathology 1996; 28: 25-32

28 Bacon CM, Diss TC, Ye H, Liu H, Goatly A, Hamoudi R. Follicular lymphoma of the thyroid gland. Am J Surg Pathol 2009; 33: 22-34 doi:10.1097/PAS.0b013e31817d7470

29 Chuang S, Lin C, Shen F, Liao P, Liao Y, Chang JH. Detecting clonal rearrangement in non-Hodgkin's lymphomas in Taiwan by polymerase chain reaction. Leuk Lymphoma 2003; 44: 117-121

30 Taheri ZM, Ziazi LM, Dorudinia A, Nadji SA, Mohammadi F. Clonality of the immunoglobulin heavy chain genes in B cell non-hodgkin lymphoma using semi-nested PCR. Tanaffos 2011; 10: 25-31

31 Medeiros LJ, Carr J. Overview of the role of molecular methods in the diagnosis of malignant lymphomas. Arch Pathol Lab Med 1999; 123: 1189-1207

32 Theriault C, Galoin S, Valmary S, Selves J, Lamant L, Roda D. PCR analysis of immunoglobulin heavy chain (IgH) and TcR-gamma chain gene rearrangements in the diagnosis of lymphoproliferative disorders: results of a study of 525 cases. Mod Pathol 2000; 13: 1269-1279

33 Saxena A, Alport EC, Moshynska O, Kanthan R, Boctor MA. Clonal B cell populations in a minority of patients with Hashimoto's thyroiditis. J Clin Pathol 2004; 57: 1258-1263

34 Matsubayashi S, Tamai H, Morita T, Fukata S, Matsuzuka F, Suzuki T. Hashimoto's thyroiditis manifesting monoclonal lymphocytic infiltration. Clin Exp Immunol 1990; 79: 170-174

35 [No authors listed]. Effect of age on the characteristics and clinical behavior of non-Hodgkin's lymphoma patients. The Non-Hodgkin's Lymphoma Classification Project. Ann Oncol 1997; 8: 973-978

$36 \mathrm{Ott} G$, Rosenwald A. Molecular pathogenesis of follicular lymphoma. Haematologica 2008; 93: 1773-1776

37 Freedman AS. Biology and management of histologic transformation of indolent lymphoma. Hematology Am Soc Hematol Educ Program 2005; 314-320

38 Zeppa P, Cozzolino I, Peluso AL, Troncone G, Lucariello A, Picardi M. Cytologic, flow cytometry, and molecular assessment of lymphoid infiltrate in fine-needle cytology samples of Hashimoto thyroiditis. Cancer 2009; 117: 174-184 doi:10.1002/cncy.20022

39 Freedman A. Follicular lymphoma: 2014 update on diagnosis and management. Am J Hematol 2014; 89: 429-436

40 Ferlito A, Robbins KT, Silver CE, Hasegawa Y, Rinaldo A. Classification of neck dissections: An evolving system. Auris Nasus Larynx 2009; 36: $127-134$ 